\title{
Antidiabetical and hypoglycemic action of mushroom polysaccharides
}

\author{
Ekaterina Antonceva ${ }^{1, *}$ and Mark Shamtsyan ${ }^{1}$ \\ ${ }^{1}$ Saint-Petersburg State Institute of Technology, 190013, Moskovsky pr., 26, Saint-Petersburg, Russia
}

\begin{abstract}
Diabetes mellitus is a group of metabolic diseases characterized by hyperglycemia caused by impaired insulin secretion, insulin action, or both. The World Health Organization identifies 4 types of diabetes: type 1 diabetes is characterized by inadequate production of insulin in the human body, type 2 is characterized by insulin resistance, impaired glucose tolerance and impaired fasting glycemia and gestational diabetes. The following criteria are important in studies of antidiabetic action: the level of glucose in the blood plasma, the level of various types of lipoproteins, the effect on immunity, the antioxidant effect, the level of insulin in the blood plasma, the effect on the intestinal microbiota, structural changes in $\beta$-cells of the pancreas glands. Many cultures of fungi are demonstrated high activity against hypoglycaemia, insulin resistance and dyslipidemia. The glucan part is often found in the composition of the investigated mushroom preparations. The $\beta$-glucans of fungi are known for their wide and varied biological activities. There is a hypothesis that it is these substances that are responsible for the antidiabetic effect of fungi. According to researchers, it is possible to identify how many possible key mechanisms of action of natural polysaccharides on diabetes mellitus: - an increase in plasma insulin levels and a decrease in the activity of pancreatic glucagon; - an increase in the sensitivity of the insulin receptor and a decrease in insulin resistance; - increased synthesis of glycogen in the liver; - improving the use of glucose by peripheral tissues; - antiinflammatory effect.
\end{abstract}

\section{Introduction}

Diabetes mellitus is a group of chronic disease characterized by a high blood glucose level, leading to systemic disruption of all types of metabolism and damage to the micro- and macrovascular system of the body. Hyperglycemia is caused by impaired insulin secretion, insulin action, or both. As a result of the diabetic process, a number of complications develop, such as blindness, renal failure, neuropathy, stroke, and myocardial infarction. There are two well-known forms of the disease: genetically determined insulin-dependent type 1 diabetes, which develops as a result of autoimmune damage to $\beta$-cells of the pancreas, and the most common type 2 insulin-resistant diabetes (approximately $90 \%$ of all cases of diabetes) [1].

*Corresponding author:_p_m_@mail.ru 


\section{Types of diabetes}

Today, the World Health Organization identifies 4 types of diabetes.

\subsection{Type 1 diabetes}

Type 1 diabetes was previously called insulin-dependent type of diabetes (juvenile) and is characterized by inadequate production of insulin in the human body, which leads to impaired glucose metabolism. Insulin is synthesized in the $\beta$-cells of the islets of Langerhans in the pancreas. This type of diabetes includes diabetes caused by the autoimmune destruction of the islets of Langerhans, which leads to absolute insulin deficiency and idiopathic diabetes. People with type 1 diabetes require daily insulin to regulate the amount of glucose in their blood. The causes of type 1 diabetes are still unclear. Symptoms include excessive urination (polyuria), thirst (polydipsia), constant hunger, weight loss, vision changes, and fatigue [2].

\subsection{Type 2 diabetes}

Of all people with diabetes, the vast majority (90-95\%) suffer from type 2 diabetes, which was previously called non-insulin-dependent diabetes or adult diabetes. This type of disease is characterized by insulin resistance and usually patients have a relative insulin deficiency [1]. At least initially, and often throughout their lives, these people do not need insulin injections to survive. Symptoms may be absent or not expressed, so the disease is difficult to diagnose in the early stages, while it has not yet caused complications. There are probably many different causes of this form of diabetes. Although the specific etiology is unknown, autoimmune $\beta$-cell destruction does not occur [2].

Most researchers identify the following factors leading to the development of type 2 diabetes: dietary disorders associated with a sedentary lifestyle and the consumption of high-calorie fatty foods with a high content of easily digestible carbohydrates $[3,4]$. According to modern studies, long-term consumption of fatty foods with a high carbohydrate content leads to a violation of the intestinal microflora, which leads to the development of "low-grade inflammation". As shown by a number of researchers, this ultimately leads to the development of insulin resistance in insulin-dependent tissues (muscles, liver and adipose tissue), in other words, to a systemic decrease in sensitivity to the anabolic hormone insulin [5].

\subsection{The third group of diabetes}

The third isolated group of diseases includes decreased glucose tolerance and impaired fasting glycemia, which are intermediate states between normal and diabetes. People with impaired glucose tolerance and impaired fasting glycemia are at high risk for type 2 diabetes, but this may not happen $[2,6]$.

\subsection{Gestational diabetes}

The fourth and last type of diabetes to be isolated is gestational diabetes, or pregnancy diabetes, characterized by increased blood glucose (hyperglycemia) that develops or is first detected during pregnancy. This diabetes is characterized by elevated glucose levels (above normal), but still lower than in other types. It is usually temporary, but it can also carry a long-term risk of developing type 2 diabetes. Gestational diabetes is diagnosed through 
prenatal screening, not symptoms. This type of diabetes affects about $7 \%$ of pregnant women $[1,2,7]$.

Diabetes is now widespread in the world, the prevalence of diabetes among people over 18 years old in 1980 was from $4.7 \%$, in 2014 it increased to $8.5 \%$ and continues to grow. The number of diabetic patients in Russia at the beginning of 2016 is $9.3 \%$ of the country's population [2].

\section{Biological activity of fungi against diabetes and hyperglycaemia}

More than 2 thousand edible or medicinal mushrooms have been identified, many of which are widely consumed due to their properties to positively affect human health. These properties are due to biologically active substances that fungi synthesize during their growth and development, including polysaccharides.

One of the most biologically active polysaccharides of fungi are the homopolysaccharides glucans, which are part of the cell walls. Fungal $\beta$-glucans are polymers of D-glucose linked by $\beta$-(1,3)-(1,6)-glycosidic bonds. These bonds are not hydrolyzed by enzymes secreted by the pancreas [5].

For the fungi Pleurotus ostreatus, Grifola frondosa, Schizophyllum commune, Sclerotium rolfsii, Lentinus edodes, Ganoderma lusidum, Hericium erunaceus and polysaccharides derived from them, a therapeutic effect has been shown against the metabolic syndrome characterized by hyperglycemia associated with diabetes, hyperglycemia associated with diabetes, obesity and hypertension [8-11].

In the case of diabetes, the following criteria are distinguished, which are important in studies of antidiabetic action: the level of glucose in the blood plasma, the level of various types of lipoproteins, the effect on immunity, the antioxidant effect, the level of insulin in the blood plasma, the effect on the intestinal microbiota, structural changes in $\beta$-cells of the pancreas glands, etc. [5].

It is believed that dysglycemia and lipid metabolism disorders are the main criteria for metabolic syndrome. These factors are very closely related. For example, diabetes often develops insulin resistance and dyslipidemia, characterized by high triglyceride levels, low high-density lipoprotein levels, and high levels of low-density lipoproteins [3,4].

Various studies have reported that mushroom polysaccharides reduce the symptoms of diseases. Antidiabetic properties in laboratory rodent studies have previously been noted for the following mushroom cultures: Agaricus brasiliensis, Agrocybe chaxingu, Catathelasma ventricosum, Grifola frondosa, Phellinus linteus, Pleurotus eryngii, Pleurotus florida, Pleurotus sajor-caju, Tremella fuciformis, Ganoderma lucidum [12, 13], Lentinus strigosus, Pleurotus tuber-regium, Sparassis crispa и Tremella aurantialba [5].

Neyrinck et al. studied the effectiveness of the fungal chitin-glucan complex in correcting gut microbiota and glucose and lipid metabolism in obese mice on a high fat diet. The chitin-glucan complex significantly reduced body weight gain, development of fat mass, glucose intolerance, hyperglycemia, and impaired fat metabolism. The use of the drug in food had a positive effect on metabolism, in particular, it slowed down the development of obesity and metabolic disorders associated with it, which was explained by the restoration of the composition and activity of the intestinal microbiota $[14,15]$. According to the authors, fungal polysaccharides, even in small quantities, have prebiotic properties due to the immunoregulatory mechanism, which suppresses the inflammatory reaction in the intestine and stimulates the growth of probiotic microflora [16-18].

Kim et al. investigated and compared water-soluble $\beta$-glucans and oligosaccharides obtained by enzymatic hydrolysis of glucans from the fungus Agaricus blazei. Both drugs showed hypoglycemic, hypotriglyceride, hypocholesterol and anti-atherosclerotic effects in 
rats with induced diabetes, and the antidiabetic activity of oligosaccharides was twice that of non-hydrolyzed $\beta$-glucans [19].

\section{Possible mechanisms of action}

The effect of freeze-dried powders from the biomass of the edible fungus Pleurotus ostreatus and P. cystidiosus on healthy volunteers and people with type 2 diabetes was evaluated. When taking the drug for a month in patients with diabetes, there was a decrease in serum glucose and an increase in insulin levels. The mechanism of hypoglycemic action, according to the authors, is associated with an increase in glucokinase activity and stimulation of insulin secretion, resulting in an increase in glucose consumption by peripheral tissues and glycogen synthesis [20].

Sohn et al. studied the effects of a combination preparation of polycan (glucan of the fungus A. pullulans) and mulberry extract (Folium mori) for the study of diabetes complicated by hepatopathy and nephropathy in rats with a streptozotocin model of diabetes. The complex preparation showed a significant reduction in hyperglycemia, hepatopathy and nephropathy. Synergistic effects in the suppression of hyperglycemia and the development of diabetes were most pronounced when the ratio of glucans to extract was 1: 4 [21].

Studies by Niwa et al. observed the effects of Agaricus blazei on the pancreas of rats with streptozotocin diabetes. Histological examination of the islets of Langerhans after a course of taking with daily oral feeding of powdered mushroom biomass during 2 months showed an increase in the mass of pancreatic $\beta$-cells [22].

Treatment with crude exopolysaccharides from a submerged culture of the fungus Lentinus strigosus resulted in a dose-dependent decrease in serum glucose levels and regeneration in the pancreatic islets of Langerhans [23]. In a study by Lee et al., Agrocybe chaxingu culture polysaccharides prevented the destruction of pancreatic $\beta$-cells in mice [24]. Li et al. studied the effect of Pleurotus eryngii extract in mice with an alloxan model of diabetes. When treated with the extract, mice showed a decrease in hyperglycemia, an increase in insulin levels in the blood, an increase in glycogen synthesis, as well as partial and gradual restoration of pancreatic $\beta$-cells damaged by alloxan [25].

Another study performed $70 \%$ partial pancreatectomy in mice to examine the effects of a glycoprotein preparation from Agaricus bisporus. The drug caused the regeneration of cells of the pancreas, which indicates that the induction of proliferation of islet $\beta$-cells has a therapeutic effect [26]. Yamac studied the effect of aqueous extracts of Agaricus bisporus biomass on rats with a streptozotocin model of diabetes. In addition to reducing hyperglycemia and significantly increasing insulin levels, there was also an unexpected effect of increasing the cellularity of the pancreatic islets of Langerhans and their apparent colonization of $\beta$-cells [27].

In recent years, researchers have paid attention to the role of the gut microbiota in glucose metabolism. For example, Cao et al. Investigated the effect of $\beta$-glucans from a culture of baker's yeast Saccharomyces cerevisiae in mice with a streptozotocin model of diabetes on a hyperlipidemic diet. In mice, liver glycogen synthesis increased and lipid accumulation decreased. The drug showed significant hypoglycemic activity, accompanied by an improvement in metabolism. Presumably, the mechanism of hypoglycemic action may be associated with a change in the intestinal microbiota [28].

According to most researchers, it is possible to identify how many possible key mechanisms of action of natural polysaccharides on diabetes mellitus:

- an increase in plasma insulin levels and a decrease in the activity of pancreatic glucagon (a decrease in the breakdown of glycogen and gluconeogenesis); 
- an increase in the sensitivity of the insulin receptor, leading to a decrease in insulin resistance;

- increased synthesis of glycogen in the liver;

- improving the use of glucose by peripheral tissues;

- anti-inflammatory effect;

- antioxidant and antiradical action [29-31].

Currently, diabetes is treated with either insulin replacement therapy or oral hypoglycemic drugs such as sulfonylurea derivatives, biguanides, thiazolidinediones, and alpha-glucosidase inhibitors. However, these drugs have a large number of undesirable side effects: they increase blood pressure, cause dry mouth, constipation, headache, heart defects and obesity. In addition, some hypoglycemic drugs, such as biguanides and sulfonylureas, have a good hypoglycemic effect and have little effect on the development of long-term complications in diabetes [32]. Therefore, the search for natural non-toxic agents capable of providing preventive and therapeutic antidiabetic action is still relevant today.

\section{Conclusion}

Diabetes is a widespread and varied disorder of glucose metabolism. The variety of factors, processes and parameters affecting glucose metabolism, interrelated and dependent on it, are very large and represent a difficult object for research. Mushroom polysaccharides have shown good results and are promising subjects for further research.

\section{References}

1. American Diabetes Association, Diabetes care 37(1), 81-S90 (2014) DOI: $10.2337 / \mathrm{dc} 14-\mathrm{S} 081$

2. World Health Organization Global Report on Diabetes (Geneva, 2016) https://apps.who.int/iris/bitstream/handle/10665/204871/9789241565257_eng.pdf

3. E.J. Cho, H.J. Hwang, S.W. Kim, J.Y. Oh, Y.M. Baek, J.W. Choi, S.H. Bae, J.W. Yun, Applied microbiology and biotechnology 75(6), 1257-1265 (2007) DOI: 10.1007/s00253-007-0972-2

4. J.Y. Yang, E. Moon, S.H. Nam, M. Friedman, Journal of agricultural and food chemistry 60(30), $7442-7449$ (2012) DOI: 10.1021/jf3017749

5. M. Friedman, Foods 5(4), 80 (2016) DOI: 10.3390/foods5040080

6. Definition, diagnosis and classification of diabetes mellitus and its complications: report of a WHO consultation, Part 1, Diagnosis and classification of diabetes mellitus (No. WHO/NCD/NCS/99.2), World Health Organization (1999) https://apps.who.int/iris/bitstream/handle/10665/66040/WHO_NCD_NCS_99.2.pdf

7. Diagnostic criteria and classification of hyperglycaemia first detected in pregnancy (No. WHO/NMH/MND/13.2), World Health Organization (2013) https://www.who.int/publications/i/item/diagnostic-criteria-and-classification-ofhyperglycaemia-first-detected-in-pregnancy

8. S. Wasser, Biomedical journal 37(6), 345-356 (2014) DOI: 10.4103/2319-4170.138318

9. C.H. Su, M.N. Lai, C.C. Lin, L.T. Ng, Applied microbiology and biotechnology 100(10), 4385-4393(2016) DOI: 10.1007/s00253-015-7260-3

10. T. Kundakovic, M. Kolundzic, Current topics in medicinal chemistry 13(21), 27342744 (2013) DOI: 10.2174/15680266113136660196 
11. M. Shamtsyan, E. Antontceva, A. Panchenko, N. Petrishchev, Hyperlipidemic and hypocholesterolic action of submerge cultured mushrooms, Journal of Hygienic Engineering and Design 7, 96-99 (2014)

12. D. Pan, D. Zhang, J. Wu, C. Chen, Z. Xu, H. Yang, P. Zhou, PloS one 8(7), 68332 (2013) DOI: 10.1371/journal.pone.0068332

13. M. Shamtsyan, Potential to develop functional food products from mushroom bioactive compounds, Journal of Hygienic Engineering and Design 15, 51-59 (2016)

14. A.M. Neyrinck, S. Possemiers, W. Verstraete, F. De Backer, P.D. Cani, N.M. Delzenne, The Journal of nutritional biochemistry 23(1), 51-59 (2012) DOI: 10.1016/j.jnutbio.2010.10.008

15. K.M.I. Bashir, J.S. Choi, International journal of molecular sciences 18(9), 1906 (2017) DOI: 10.3390/ijms18091906

16. C.J. Chang, C.S. Lin, C.C. Lu, J. Martel, Y.F. Ko, D.M. Ojcius, S.F. Tseng, T.R. Wu, Y.Y.M. Chen, J.D. Young, H.C. Lai, Nature communications 6(1), 1-19 (2015) DOI: 10.1038/ncomms8489

17. M. Jayachandran, J. Xiao, B. Xu, International journal of molecular sciences 18(9), 1934, 1-12 (2017) DOI: 10.3390/ijms18091934

18. E. Antontceva, S. Sorokin, M. Shamtsyan, L. Krasnikova, Influence of Pleurotus ostreatus preparations on fermentation products of lactic acid cultures, Journal of Hygienic Engineering and Design 22 (2018)

19. Y.W. Kim, K.H. Kim, H.J. Choi, D.S. Lee, Biotechnology letters 27(7), 483-487 (2005) DOI: 10.1007/s10529-005-2225-8

20. W.B.N. Jayasuriya, C.A. Wanigatunge, G.H. Fernando, D.T.U. Abeytunga, T.S. Suresh, Phytotherapy research 29(2), 303-309 (2015) DOI: 10.1002/ptr.5255

21. J.H. Sohn, J.W. Kim, G.W. Jung, D.C. Park, S.B. Moon, H.R. Cho, S.K. Ku, J.S. Choi, Current Nutrition \& Food Science 14(3), 235-246 (2018) DOI: $10.2174 / 1573401313666170609094842$

22. A. Niwa, T. Tajiri, H. Higashino, Journal of clinical biochemistry and nutrition 48(3), 194-202 (2011) DOI: 10.3164/jcbn.10-78

23. M. Yamac, G. Kanbak, M. Zeytinoglu, G. Bayramoglu, H. Senturk, M. Uyanoglu, Journal of medicinal food 11(3), 513-517 (2008) DOI: 10.1089/jmf.2007.0551

24. B.R. Lee, Y.P. Lee, D.W. Kim, H.Y. Song, K.Y. Yoo, M.H. Won, T.C. Kang, K.J. Lee, K.H. Kim, J.H. Joo, H.J. Ham, J.H. Hur, S.W. Cho, K.H. Han, K.S. Lee, J. Park, W.S. Eum, S.Y. Choi, Molecules and Cells 29(4), 349-354 (2010) DOI: 10.1007/s10059-010-0044-9

25. J.P. Li, Y.L. Lei, H. Zhan, International journal of medicinal mushrooms 16(3), 219225 (2014) DOI: 10.1615/IntJMedMushr.v16.i3.20

26. Y. Wang, Y. Liu, H. Wang, C. Li, P. Qi, J. Bao, Experimental Biology and Medicine 237(3), 287-296 (2012) DOI: 10.1258/ebm.2011.011251

27. M. Yamac, G. Kanbak, M. Zeytinoglu, H. Senturk, G. Bayramoglu, A. Dokumacioglu, L.J.L.D. Van Griensven, International Journal of Medicinal Mushrooms 12(4), 379389 (2010) DOI: 10.1615/IntJMedMushr.v12.i4.50

28. Y. Cao, S. Zou, H. Xu, M. Li, Z. Tong, M. Xu, X. Xu, Molecular nutrition \& food research 60(12), 2678-2690 (2016) DOI: 10.1002/mnfr.201600032

29. P.C. Wang, S. Zhao, B.Y. Yang, Q.H. Wang, H.X. Kuang, Carbohydrate polymers 148, 86-97 (2016) DOI: 10.1016/j.carbpol.2016.02.060 
30. T. Vitak, B. Yurkiv, S. Wasser, E. Nevo, N. Sybirna, World journal of diabetes 8(5), 187-201 (2017) DOI: 10.4239/wjd.v8.i5.187

31. A.T. Lima, M.N. Santos, L.A. de Souza, T.S. Pinheiro, A.A. Paiva, C.M. Dore, M.S. Costa, N.D. Santos, Y.G. Baseia, R.M. Araújo, E.L. Leite, Carbohydrate polymers 144, 400-409 (2016) DOI: 10.1016/j.carbpol.2016.02.050

32. B. Luna, M.N. Feinglos, Oral agents in the management of type 2 diabetes mellitus, American family physician 63(9), 1747-1756 (2001) PMID: 11352285 